\title{
Analysis On The Trends And Evolution of Aerobic Exercise Science Research In The United States Based on Knowledge Atlas
}

Jian Li

Shaanxi University of Science and Technology

Chong Zhang

Xi'an Physical Education University

Meiyue Li (D13451974@qq.com )

Xi'an Medical University

\section{Research Article}

Keywords: Aerobic Exercise, research trends, visualization, CiteSpace V

Posted Date: September 24th, 2021

DOI: https://doi.org/10.21203/rs.3.rs-902626/v1

License: (c) This work is licensed under a Creative Commons Attribution 4.0 International License.

Read Full License 


\section{Abstract}

\section{Background}

Aerobic exercise is an effective physical exercise to promote human health and prevent diseases. It has been the focus of attention from all walks of life. Only by effectively grasping the key documents can we enter the forefront of relevant research fields.

\section{Methods}

Using the Web of Science ${ }^{\mathrm{TM}}$ core collection, 1,833 documents from the American aerobic exercise studies, which conducted scientific measurement and visual analysis using the CiteSpace? analysis software

\section{Results}

American aerobic research development of polynomial function growth, the main research focus in the university research institutions, formed the sports science dominated multidisciplinary group, research mainly focus on the cognitive improvement and heart function of cardio, aerobic exercise training and health, exercise risk, body composition and body load in the evolution of body load. In recent years, aerobic exercise research has been introduced to enhance human health, in the process of developing cardiovascular disease, strength training, exercise intensity, weight control, human inflammation, insulin resistance, metabolic syndrome, randomized controlled experiments and resistance resistance training gradually evolved into a new trend of aerobic exercise research in the United States.

\section{Conclusions}

Aerobic exercise research has been introduced to enhance human health, in the process of developing cardiovascular disease, strength training, exercise intensity, weight control, human inflammation, insulin resistance, metabolic syndrome, randomized controlled experiments and resistance resistance training gradually evolved into a new trend of aerobic exercise research in the United States.

\section{Background}

Aerobic exercise is an effective physical exercise to promote human health and prevent diseases. It has been the focus of attention from all walks of life. What are the characteristics and trends of current aerobic exercise research in the United States? What are the research hot spots? Facing the massive scientific and technical information literature, we only effectively grasps the key literature, can enter the correlation research domain frontier. By searching CNKI database, the author found that there were 3774 articles on aerobic exercise research in China since 1980, and 1134 articles were published in Chinese core journals, which showed that Chinese scholars have paid considerable attention to the scientific research on aerobic exercise. 
In view of this, this paper attempts to use the CiteSpace $\otimes$ scientific literature analysis tool to analyze the relevant research literature on aerobic exercise, which is included in the Web of science ${ }^{\mathrm{Tm}}$ core set database. This paper makes Bibliometrics and visualization analysis from the aspects of literature quantity, organization distribution, discipline distribution, core author distribution, research hot spot and development trend, etc., the relevant information contained in the literature of aerobic exercise research included by Web of science database can be extracted to understand the research ideas and research trends of American scholars, so as to identify and master the research trends in the field of aerobic exercise in the United States. This paper reveals the development of knowledge and discipline of aerobic exercise research, and provides a new perspective for mastering and studying aerobic exercise research in the United States.To expand the research horizons of relevant scholars on aerobic exercise, draw lessons from high-level research methods, and help the development of Chinese sports, so as to realize the goal of China from a big sports country to a strong sports country as soon as possible.

\section{Data Sources And Research Methods}

\subsection{Data sources}

In this study, SCIE, SSCl and A \& $\mathrm{HCl}$ of the Web of Science database were used as the source databases for literature retrieval,the retrieval formula is shown in Table 1.Identified retrieval strategy bits:topic="aerobic sport"or"aerobic exercise"or"aerobic activity"or"aerobic training", literature type selection Article, time span from January 1,2007 to May 13, 2021, a total of 1833 related literatures were retrieved, and 1771 literatures were selected from English, which were used as the data set of this study.

Table 1 Literature sources of this study

Project

Literature sources

Retrieve the way

Retrieve subject words

Retrieve the fixed number of year

Category

Document Type

Language

The literature number
Literature retrieval

Web of Science core collection

Subject retrieval form

TS=("aerobic sport"or" aerobic exercise" or" aerobic activity" or"aerobic training")

(2007-01-01-2021-05-13)

\subsection{Methodology}


With the help of the scientific literature analysis tool of CiteSpace V developed by Dr. Chen Chaomei of

Drexel University [23], 1,771 papers were processed. In order to analyze the development trend of aerobic exercise, the methods of quantitative and visual analysis, such as text volume, organization, Country Distribution, core author analysis, core keyword analysis, co-citation analysis, co-occurrence network analysis, etc. To analyze the development trend of aerobic exercise research in the United States, grasp the research trends and hot spots in the field of aerobic exercise.

\subsection{Research procedures}

This paper analyzes the visual knowledge map of aerobic exercise by using the software "Cite Space V", which mainly includes four parts: theme determination, data preparation, software analysis setting and visual knowledge map analysis.

(1) Subject determination. This study refers to the translation methods of many works and academic papers, and consults many experts in the field of sports science and translation, using "aerobic sport"or"aerobic exercise"or"aerobic activity"or"aerobic training" as retrieval subject words.

(2) Data preparation. The 1883 articles retrieved by WOS database were extracted, and 1771 articles were selected by eliminating the repetitive and unrelated articles.

(3) Software analysis settings. In "CiteSpace", the time is set from 2007-01-01 to 2021-05-13, and the time slice is one year as a segment. When analyzing countries (regions), authors and disciplines, the threshold term is "TopN = 50", and the algorithm does not cut out. When analyzing and researching hot keywords, the threshold item is "TopN\%=50\%", the algorithm is "critical path calculation method", and the cutting method is "overall network pruning".

(4) Visual knowledge map analysis. After we get the graph, we can see the multi-information of each node. In addition, the introduced time distribution can be judged by the color of each tree ring, and the knowledge flow between clusters can also be seen from time.

\section{Results And Analysis}

\subsection{Annual distribution of aerobic exercise research literature}

The time series change of the number of scientific research papers is an important index to measure the development of a certain field. It is of great significance to evaluate the stage of this field and to predict the dynamic trend of development. In 2007, the Web of Science ${ }^{T M}$ core collection included aerobic exercise research literature with a total of 111 articles. Among them, the development was very slow from 2007 to 2009 , with 305 papers published, accounting for $16.78 \%$ of the total literature with an annual average of 102 papers; since 2009 (110 papers), the number of papers published has increased rapidly, increasing by 
30.95\% compared with 2008. This study has collected the literatures collected in recent 15 years. Compared with the development of aerobic exercise, 15 years is not a long time, but it can be seen from Fig. 1 that the number of articles on aerobic exercise research in the United States is increasing.

\subsection{Institutional distribution of aerobic exercise research in the United States}

The analysis of American aerobics exercise research institutions can effectively grasp the level of scientific research, scientific and technological strength and the distribution of scientific research results.

Through the co-occurrence analysis function of the research unit of the CITESPACE V analysis software, the co-occurrence genealogy of the major research institutions of aerobic sports in the United States is obtained. As shown in Fig. 2, the main research institutions of aerobic exercise in the United States are closely connected and form a network, forming a large aerobic exercise research system. The close contact between the related research institutions is beneficial to the communication among the research institutions, ensuring the systematicness and continuity of the related research direction, and promoting the continuous progress of the research. As can be seen from Table 1, the major research institutions of aerobic exercise in the United States are concentrated in institutions of higher learning, and all of the top 10 institutions in terms of the number of articles published are from institutions of higher learning. Because the American aerobic exercise research system is based on the university platform, it is beneficial to the combination of aerobic exercise research and university research system, and to promote the development of aerobic exercise research system. This is why most of the participants in the aerobic exercise study came from the university system.

Table 2 lists that the sports from 2007 to 2021 are the top 10 institutions, all of which come from institutions of higher learning. Univ Illinois, Univ N Carolina and Univ Wisconsin ranked in the top three, with 73 papers, 53 papers and 51 papers respectively, followed by Univ Texas Austin, Univ Colorado, Univ Pittsburgh, Univ S Carolina, Univ Michigan, Ball State Univ and Univ Connecticut. By analyzing the published articles of Univ Illinois, it is found that Univ Illinois has a multi-disciplinary research system, and the integration of multi-disciplines provides an important platform for the research of aerobic exercise. His team is mainly engaged in the research of sport sciences $\square$ physiology $\square$ nutrition $\square$ dietetics $\square$ psychology $\llbracket$ rehabilitation $\square$ hospitality leisure sport tourism $\square$ psychology applied and gerontology. 
Table 2

top 10 institutions in U.S. Aerobic Exercise Research

\begin{tabular}{|llll|}
\hline Rank & Organization & Amount of writing & Centrality \\
\hline 1 & Univ Illinois & 73 & 0.14 \\
\hline 2 & Univ N Carolina & 53 & 0.09 \\
\hline 3 & Univ Wisconsin & 51 & 0.02 \\
\hline 4 & Univ Texas Austin & 42 & 0.15 \\
\hline 5 & Univ Colorado & 40 & 0.06 \\
\hline 6 & Univb Pittsburg & 35 & 0.09 \\
\hline 7 & Univ S Carolina & 33 & 0.11 \\
\hline 8 & Univ Michigan & 33 & 0.03 \\
\hline 9 & Ball State Univ & 32 & 0.05 \\
\hline 10 & Univ Connecticut & 29 & 0.01 \\
\hline
\end{tabular}

\subsection{Distribution of major disciplines in aerobic exercise research in the United States.}

This paper uses the CiteSpace $V$ analysis software to categorize the aerobic exercise research in the United States, covering 26 disciplines in total high frequency subjects, including sport sciences(1818)

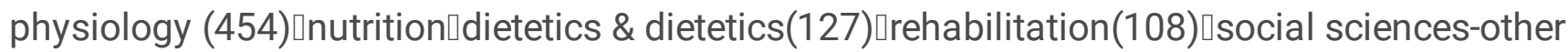

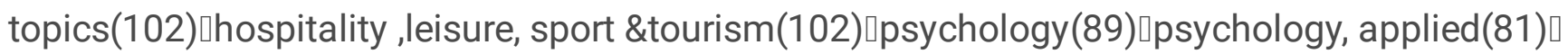
orthopedics(47) $\square$ pediatrics(42), etc.. As can be seen from Fig. 3, sport sciences (1.48) had the highest centrality, which is followed by psychology $(0.07)$, orthopedics $(0.07)$, psychology $(0.04)$, social sciencesother topics (0.01), hospitality, sport \& tourism (0.01), general \& internal medicine (0.01), public, \& occupational health (0.01), and neurosciences \& neurology (0.01). This shows that the United States in the field of aerobic exercise to form a multi-disciplinary interdisciplinary sports science-led, aerobic exercise research from the theory and methods to provide a solid foundation.

\subsection{Core author analysis of aerobic exercise research in the United States}

Through the co-occurrence analysis function of CiteSpace $V$ analysis software, a co-occurrence pedigree of American aerobic exercise researchers is obtained, as shown in Fig. 4. An analysis of the co-occurrence genealogy of the literature shows that the connections among aerobic exercise researchers in the United States are crisscrossed, indicating that the authors are closely related to each other. The whole aerobic 
exercise researchers are connected with each other to form a large-scale research system, which makes the research on aerobic exercise more systematic and open, and improves the research efficiency.

As shown in Table 3, according to the amount of papers published by high-yield authors, Dr. Fernhall is a professor at the University of Illinois in the United States, and has published the most papers with 22 papers. His main research fields are physical exercise of mentally retarded teenagers, the influence of aerobic exercise on blood pressure changes of different races, the physiological response of super exercise, the catecholamine response of patients with Down's syndrome to the maximum exercise, and the future development of sports, etc.. ${ }^{[1]}$ Professor Stout JR of University of Central Florida has published 21 articles, ranking second. The main research areas are cardiovascular, body composition, strength, aerobic capacity, hormones, nutritional supplement, maximum oxygen uptake, resistance training, fatigue and recovery, etc. ${ }^{[2]}$ Dr. Foster $\mathrm{C}$ is a professor at the university of Wisconsin Medical School, and has published 20 articles, ranking third, mainly engaged in high-intensity interval training, aerobic exercise ability, maximum oxygen uptake, altitude training, etc.. ${ }^{[3]}$ The research team centered on Dr. Steven $\mathrm{N}$ Blair, a professor at the university of South Carolina, mainly focuses on cardiopulmonary endurance, physical activity, heart rate, physical exercise, public health, diseases and other fields. Professor Hillman $\mathrm{CH}$ is mainly engaged in the research of children's physical activity, health and cognitive function. ${ }^{[5]} \mathrm{Mr}$. Fukuda DH of Fitchburg State University, Mr. Nindl BC of University of Pittsburgh, Mr. Kendall KL of Edith Cowan University, Mr. Smith AE of University of Oklahoma and Mr. Trappe TA of Ball State University also have great influence in the field of aerobic exercise research in the United States, mainly in sports science, physiology, nutrition, rehabilitation and other academic fields, and have made outstanding contributions to the in-depth research and sustainable development of aerobic exercise in the United States.

It's worth noting that two of the top 10 most prolific authors, Fernhall $\mathrm{B}$ and Hillman $\mathrm{CH}$, are from the University of Illinois. This shows that the university has an excellent aerobic exercise research team, and has made outstanding contributions for the United States in aerobic exercise field in-depth research and sustainable development. 
Table 3

top 10 authors in U.S. aerobic exercise research

\begin{tabular}{|llll|}
\hline Ranking & Author & Institution & number of published papers \\
\hline 1 & Fernhall B & University of Illinois & 22 \\
\hline 2 & Stout JR & University of Central Florida & 21 \\
\hline 3 & Foster C & University of Wisconsin Medical School & 20 \\
\hline 4 & Blair SN & Louisiana State University & 19 \\
\hline 5 & Hillman CH & University of Illinois & 18 \\
\hline 6 & Fukuda DH & Fitchburg State University & 16 \\
\hline 6 & Nindl BC & University of Pittsburgh & 16 \\
\hline 8 & Kendall KL & Edith Cowan University & 13 \\
\hline 9 & Smith AE & University of Oklahoma & 10 \\
\hline 10 & Trappe TA & Ball State University & 9 \\
\hline
\end{tabular}

Combining the units and institutions of authors, Univ Illinois was found to be the most important aerobic exercise research institution.

\subsection{Keyword analysis of the literature on exercise as good medicine in the United States}

With CiteSpaceV software, the Node Types select key-words, the threshold is set to Top20, and the pathfinder algorithm is used to get the clustering network map of Key-word in the field of sports as good medicine in the United States (Fig. 5). It can be seen from Fig. 5 that the hot areas of aerobic exercise research in the United States mainly focus on enhancing people's health, and the research hotspots mainly focus on aerobic exercise, exercise, rehabilitation, diet, exertion, pressure, weight control, energy consumption, blood pressure, heart rate, maximum oxygen uptake and so on; The research objects mainly involve adults, children and other groups, to explore their skeletal muscle, body composition, obesity, blood, diseases and other fields; the main research methods are randomized controlled experiments and resistance training, etc. to explore the influence of different sports intensity and strength training strategies on competitive performance and human health and to further explore the exercise methods and strategies to improve human health.

\subsection{Time zone analysis of key words in American aerobic exercise literature}


This paper uses CiteSpace $V$ to draw the time zone map of key words in American aerobic exercise research (Fig. 6). Because this study collected nearly 15 years of literature, relative to the development of aerobic exercise field, 15 years is not long, but from Fig. 6 can see that the content of aerobic exercise in the United States in the last 15 years have some changes. Almost all of the research topics can be found in 2010 and before, indicating that aerobic exercise research in 2010 and before has been more comprehensive.

By analyzing Fig. 6, combing the annual high-frequency keywords and reading the American literature on aerobic exercise, we can see that the focus of American aerobic exercise research has gradually changed from aerobic exercise and physical activity to the transition of the research object of aerobic exercise. Women, men, athletes, children, teenagers, adult, runner and old people have become the main research groups. With the passage of time, the research focus of aerobic exercise has shifted to the field of physiology. From the research of skeletal muscle, body composition, heart rate and other fields to blood pressure, blood flow, plasma, oxidative stress, weight control, insulin resistance, adipose tissue, gene expression, metabolic syndrome and other fields; at the same time, the training methods have evolved alternately from resistance exercise to cardio-pulmonary fitness, resistance training, exercise intensity, etc. Among them, randomized controlled experiments and meta-analysis methods have gradually evolved into hot research methods of aerobic exercise. In addition, health, obesity, rehabilitation, quality of life, overweight and disease are also hot topics in the evolution of aerobic exercise research.

\subsection{Co-citation analysis of aerobic exercise research literature in the United States}

This paper uses CiteSpaoe $V$ to draw the co-citation map of aerobic exercise research literature in the United States. The cluster in Fig. 7 is a collection of influential and innovative classical literature on aerobic exercise research in the United States, and the key nodal literature is the evolutionary direction of aerobic exercise research in the United States. According to the clustering features of Fig. 7 and reading the related literature of aerobic exercise research, it can be divided into four knowledge clusters, each of which corresponds to a research direction of aerobic exercise research in the United States, and analyzes the key high-frequency and high-center literatures to can reveal the evolution of international aerobic exercise research path back.

\subsubsection{Knowledge cluster I: research on aerobic exercise improving cognitive ability}

In this cluster, the effect of aerobic exercise on cognitive ability should be explored. The most frequently cited literature is The effects of acute exercise on cognitive performance: a meta-analysis ${ }^{[6]}$, a metaanalysis of existing literature on acute exercise and cognitive performance, to explore the effects of exercise time, exercise intensity, type of cognitive performance evaluation and health status of 
participants on cognitive performance. The results show that exercise-induced physiological changes affect cognitive function, and the same conclusion is found in aerobic exercise studies. However, the authors point out that acute exercise generally has a small effect on cognitive performance and may have a greater effect on specific cognitive outcomes when specific exercise parameters are used. A 2016 JE ${ }^{[7]}$ study found a positive association between PA, health, cognition and academic achievement. The effects of PA on many cognitive factors remain to be explored, such as type of exercise, amount of exercise, frequency and duration of exercise. The results of the 2017 Anderson-Hanley $C^{[8]}$ showed that it is possible for older adults to use a novel, theoretically derived neuromotor game, and provide promising new evidence, neuromotor gaming has been shown to produce greater cognitive benefits than any of its components. Results demonstrate feasibility for older adults to use a novel and theologically-derived neuro-erotic game, and also provide promising new evidence that neuro-exergaming can yield greater cognitive benefit than either of its component parts. The 2017 Kao SC ${ }^{[9]}$ study showed that there were significant differences between healthy aerobic and muscular exercise and working memory and academic achievement. Since most studies focus on the health benefits of aerobic fitness for children, this study shows the importance of muscle health for cognitive health before puberty. In 2019, MoraGonzalez $\mathrm{J}^{[10]}$ studied the relationship between physical health, sedentary time and physical activity (PA) and working memory and neural electrical activity in overweight children, concluded that the relationship between PA and working memory is intensity dependent. The 2019 Syväoja HJ ${ }^{[11]}$ study showed that the changes of aerobic and muscular fitness were positively correlated with the changes of academic performance, but the changes of motor skills were only marginal significant. However, better motor skills (although not systematically) independently predicted better academic achievement a year later, while aerobic exercise and muscle health were not. Better academic achievement predicts better motor skills, aerobic fitness, and muscle fitness. Developmental changes in adolescence may lead to parallel and simultaneous changes in academic achievement and PF.

\subsubsection{Knowledge Cluster II: aerobic exercise improves cardiac function}

In knowledge clustering II, McDaniel BB ${ }^{[12]}$ suggests that water sports may be more tolerable for sedentary overweight people than land sports. By high-intensity interval training 11 college students in water for five weeks, it was found that their heart, lung health and body composition were significantly improved. In 2019, Brito LC ${ }^{[13]}$ compared the effects of morning and evening aerobic training on blood pressure, finding that evening aerobic training reduces clinical and ambulatory blood pressure because of reducing systemic vascular resistance and sympathetic nervous system regulation, suggesting that evening training (ET) may have a greater effect on blood pressure. Gormley SE ${ }^{[14]}$ research shows that high-intensity aerobic exercise improves V02max in healthy young people more than low-intensity aerobic exercise when the amount of exercise is controlled. By evaluating the effects of simultaneous strength and aerobic endurance training on cardiovascular and cardiorespiratory fitness in college athletes, studies have shown that synchronized training enhances cardiovascular and cardiorespiratory 
function in athletes. In addition, authors such as Boudreaux BD, Siddhartha S Angadi, Scott Trappe and Nilsen TS have made in-depth researches in areas such as long-term adherence to aerobic exercise can improve the body's resistance and enhance the heart and lung function.

\subsubsection{Knowledge clustering III: research on aerobic exercise training and health}

With the continuous development of aerobic exercise research in the United States, the research on aerobic exercise training and health promotion has become the focus of scholars. Long-term aerobic exercise can increase the amount of hemoglobin in the body, improve body resistance, anti-aging, enhance the efficiency of the cerebral cortex and heart and lung function, increase fat consumption, prevent arteriosclerosis and reduce the incidence of cerebrovascular disease as well as other effects. Nelson ME ${ }^{[16]}$ suggests older people take aerobic intensity exercise to maintain or increase flexibility, and some others who are at risk of falling take balance exercise. In addition, older persons should have an activity plan to achieve recommended physical activity, combined with prevention and treatment recommendations. The study concluded that promotion of physical activity in the elderly should emphasize moderate intensity aerobic activity, increased muscle activity, reduced sedentary behavior and risk management; ${ }^{[17]}$ the study of aerobic exercise found that moderate intensity exercise of more than 150 minutes per week can improve the health of overweight and obese patients. Long-term physical activity of 150-250 minutes per week is an effective method to control weight gain. Studies have also shown that aerobic endurance or resistance training can not only prevent or reduce the risk of chronic disease, but also improve human health. In 2014, Taylor-Piliae RE ${ }^{[18]}$ explored the effects of 12-week taijiquan (TC) intervention on body function and quality of life. Research shows that 12-week taijiquan intervention is more effective than routine nursing intervention in reducing the rate of falls. The results of the Ellingson LD ${ }^{[19]}$ showed that suggestions of physical activity can improve physical strength and fatigue even when combined with a sedentary lifestyle. Among women who did not meet the physical activity recommendations, women who spent less time sitting had significantly lower fatigue levels than their sedentary peers. In addition, less sitting was associated with lower levels of fatigue in women who were less active. In 2014, Madden D ${ }^{[20]}$ investigated differences in aerobic (EX) and non-motor controlled diving (CON) responses before scuba diving, and further assessed the potential relationship between venous gas embolism (VGE) and particulate (MP), we hypothesized that exercise would alter the quantity and subtype of annexin V-positive MP and VGE. 2019 McMillan AG ${ }^{[21]}$ explores the effects of exercise during pregnancy on neuromotor development in 1-month-old offspring, suggesting that exercise during pregnancy can positively affect the developmental system and improve neuromotor development, making babies more athletic. Since physical activity is an adjustable risk factor for childhood obesity, these findings suggest that exercise during pregnancy may potentially reduce the risk of childhood obesity. 


\subsubsection{Knowledge Cluster V: research on aerobic training and exercise risk, body composition and body load}

Knowledge cluster $\mathrm{V}$ is a key research document on the evolution of sports risk, body composition and body load. Hillman $\mathrm{CH}^{[22]}$ 's research on human and non-human animals shows that aerobic exercise has a positive impact on cognition and brain function, and also has a greater impact on molecular, cellular, systematic and behavioral levels, which is also helpful to improve people's physical and mental health. Physical activity and public health: updated recommendation for adults from the American college of sports medicine and the American heart association published by Haskell WL ${ }^{[23]}$ is a more detailed suggestion on body load in the process of exploration and evolution of "how much is reasonable" in the arrangement of body load dose. It is suggested that adults should ensure moderate-intensity physical exercise for 5 days a week and more than 30 minutes a day, or ensure high-intensity physical exercise for 3 days every week and more than 20 minutes every day. In addition, it is suggested that adults should carry out weightlifting exercises to exercise the strength and endurance of major muscle groups and neuromotor exercises involving balance, agility and coordination. For the elderly over 65 years old, it is suggested that they consider strength exercises to increase muscle strength, to prevent accidental falls, and to enhance flexibility and balance. This document is the most influential document in the evolution of American sports guide from 1970 to 2010 . This study undoubtedly provides a mathematical and statistical basis for further and extensive research in the field of aerobic exercise in the United States. In 2012, Hornbuckle LM $^{[24]}$ evaluated the effects of walking (W) and walking plus resistance training (WRT) on cardiovascular diseases of middle-aged African American women. Studies have shown that 12-week walking plus resistance training is more effective than walking in improving body composition and blood sugar control. Walking plus resistance training may be an important supplement to lifestyle intervention. In 2017, the research of Racette $\mathrm{SB}^{[25]}$ showed that the effect of calorie restriction on aerobic capacity and muscle strength of healthy, normal-weight, slightly overweight women and men for two years had no obvious change. Howell $\mathrm{DR}^{[26]}$ research shows that aerobic exercise plays an important role in the treatment of exercise-related concussion.

\section{Conclusion}

4.1 The research on aerobic exercise in the United States is closely related to universities, forming a university-based research system. All the top 10 high-yield research institutions are universities. Because the research system in the field of aerobic exercise in America is attached to the school system, the research problems in the field of aerobic exercise in America come directly from the school. Through the analysis of the research contents, it is found that the University of Illinois has become the most outstanding research institution in the field of aerobic sports, and its research direction has basically led the research trend of aerobic sports in the United States.

4.2 The core authors with great academic influence in the field of aerobic exercise in the United States, mainly including Fernhall B, Stout Jr, Foster C, Blair SN and Hillman CH, have exerted a profound 
influence on the in-depth research and sustainable development of aerobic exercise in the United States. In addition, the researchers in the field of aerobic exercise in the United States have formed a national aerobic exercise research system, which is conducive to systematic and in-depth study, and improve the efficiency of research. The next step is to strengthen the construction of international academic team, which is more beneficial to explore the frontier field of aerobic sports research, expand the influence of aerobic sports in the international academic community and the output capacity of scientific research results.

4.3 Research topics in the field of aerobic exercise in the United States focus on the improvement of exercise cognition and heart function, physical activity and health, exercise risk, body composition and the evolution of body load. Cardiovascular disease, cancer, strength training, exercise intensity, weight control, insulin resistance, metabolic syndrome, randomized controlled trials, and resistance training have become new trends in aerobic exercise research in the United States.

\section{Declarations}

\section{Acknowledgements}

We thank the undergraduate students who took part in the study.

\section{Authors' contributions}

LJ collected the literature and determined the analysis framework, analyzed the data, and wrote the paper. LMY and ZC managed data collection and cleaning. All authors reviewed and approved the final draft.

\section{Availability of data and materials}

The datasets used and/or analysed during the current study available from the corresponding author on reasonable request.

\section{Ethics approval and consent to participate}

This research was approved by the Ethics Committee of the Sports Department of Shaanxi University of Science and Technology. All students invited to participate were voluntary accepted to take part in the study after signing written informed consent.

\section{Funding}

Not applicable.

\section{Consent for publication}

Not applicable.

\section{Competing interests}


The authors declare that they have no competing interests.

Ethical Statement

Ethical approval for this study was granted by the Shaanxi University of Science and Technology Ethics Committee. All participants provided informed consent.

Conflict of Interest

No conflict of interest has been declared by the authors. Beyond the absence of any conflict of interest, that the manuscript was built based on the IJSM ethical standards.

\section{References}

1. Fernhall B, Borghi SA, Babu AS. The future of physical activity research: funding, opportunities and challenges. Prog Cardiovasc Dis 2015;57(4):299-305.

2. Rossow LM, Fukuda DH, Fahs CA, et al. Natural bodybuilding competition preparation and recovery: a 12-month case study. Int J Sports Physiol Perform 2013;8(5):82-92.

3. Foster C, Farland CV, Guidotti F, et al. The Effects of High Intensity Interval Training vs Steady State Training on Aerobic and Anaerobic Capacity. J Sports Sci Med 2015;14(4):747-55.

4. Lee IM, Shiroma EJ, Lobelo F, et al. Effect of physical inactivity on major non-communicable diseases worldwide: an analysis of burden of disease and life expectancy. Lancet 2012;380(9838):219-29.

5. Donnelly JE, Hillman $\mathrm{CH}$, Castelli D, et al. Physical Activity, Fitness, Cognitive Function, and Academic Achievement in Children: A Systematic Review. Med Sci Sports Exerc 2016;48(6):1223-4.

6. Chang YK, Labban JD, Gapin JI, et al. The effects of acute exercise on cognitive performance: a meta-analysis. Brain Res 2012;1453:87-101.

7. Donnelly JE, Hillman $\mathrm{CH}$, Castelli $\mathrm{D}$, et al. Physical Activity, Fitness, Cognitive Function, and Academic Achievement in Children: A Systematic Review. Med Sci Sports Exerc 2016;48(6):1223-4.

8. Anderson-Hanley C, Maloney M, Barcelos N, et al. Neuropsychological Benefits of Neuro-Exergaming for Older Adults: A Pilot Study of an Interactive Physical and Cognitive Exercise System (iPACES). J Aging Phys Act 2017;25(1):73-83.

9. Kao SC, Westfall DR, Parks AC, et al. Muscular and Aerobic Fitness, Working Memory, and Academic Achievement in Children. Med Sci Sports Exerc 2017;49(3):500-08.

10. Mora-Gonzalez J, Esteban-Cornejo I, Cadenas-Sanchez C, et al. Fitness, physical activity, working memory, and neuroelectric activity in children with overweight/obesity. Scand J Med Sci Sports 2019;29(9):1352-63.

11. Syväoja HJ, Kankaanpää A, Joensuu L, et al. The Longitudinal Associations of Fitness and Motor Skills with Academic Achievement. Med Sci Sports Exerc 2019;51(10):2050-57. 
12. McDaniel BB, Naquin MR, Sirikul B, et al. Five Weeks of Aquatic-Calisthenic High Intensity Interval Training Improves Cardiorespiratory Fitness and Body Composition in Sedentary Young Adults. J Sports Sci Med 2020;19(1):187-94.

13. Brito LC, Peçanha T, Fecchio RY, et al. Morning versus Evening Aerobic Training Effects on Blood Pressure in Treated Hypertension. Med Sci Sports Exer 2019;51(4):653-62.

14. Gormley SE, Swain DP, High R, et al. Effect of intensity of aerobic training on VO2max. Med Sci Sports Exerc 2008;40(7):1336-43.

15. Davis WJ, Wood DT, Andrews RG, et al. Concurrent training enhances athletes' cardiovascular and cardiorespiratory measures. J Strength Cond Res 2008;22(5):1503-14.

16. Nelson ME, Rejeski WJ, Blair SN, et al. Physical activity and public health in older adults: recommendation from the American College of Sports Medicine and the American Heart Association. Circulation. 2007;116(9):1094-105.

17. Donnelly JE, Blair SN, Jakicic JM, et al. Appropriate physical activity intervention strategies for weight loss and prevention of weight regain for adults. Med Sci Sports Exerc 2009;41(2):459-71.

18. Taylor-Piliae RE, Hoke TM, Hepworth JT, et al. Effect of Tai Chi on physical function, fall rates and quality of life among older stroke survivors. Arch Phys Med Rehabil 2014;95(5):816-24.

19. Ellingson LD, Kuffel AE, Vack NJ, et al. Active and sedentary behaviors influence feelings of energy and fatigue in women. Med Sci Sports Exerc 2014;46(1):192-200.

20. Madden D, Thom SR, Milovanova TN, et al. Exercise before scuba diving ameliorates decompressioninduced neutrophil activation. Med Sci Sports Exerc 2014;46(10):1928-35.

21. McMillan AG, May LE, Gaines GG, et al. Effects of Aerobic Exercise during Pregnancy on 1-Month Infant Neuromotor Skills. Med Sci Sports Exerc 2019;51(8):1671-76.

22. Hillman $\mathrm{CH}$, Erickson $\mathrm{KI}, \mathrm{Kramer} \mathrm{AF}$. Be smart, exercise your heart: exercise effects on brain and cognition. Nat Rev Neurosci 2008;9(1):58-65.

23. Haskell WL, Lee IM, Pate RR, et al. Physical activity and public health: updated recommendation for adults from the American College of Sports Medicine and the American Heart Association. Circulation 2007;116(9):1081-93.

24. Hornbuckle LM, Liu PY, llich JZ, et al. Effects of resistance training and walking on cardiovascular disease risk in African-American women. Med Sci Sports Exerc 2012;44(3):525-33.

25. Racette SB, Rochon J, Uhrich ML, et al. Effects of Two Years of Calorie Restriction on Aerobic Capacity and Muscle Strength. Med Sci Sports Exerc 2017;49(11):2240-9.

26. Howell DR, Taylor JA, Tan CO, et al. The Role of Aerobic Exercise in Reducing Persistent Sport-related Concussion Symptoms. Med Sci Sports Exerc 2019;51(4):647-52.

\section{Figures}


180

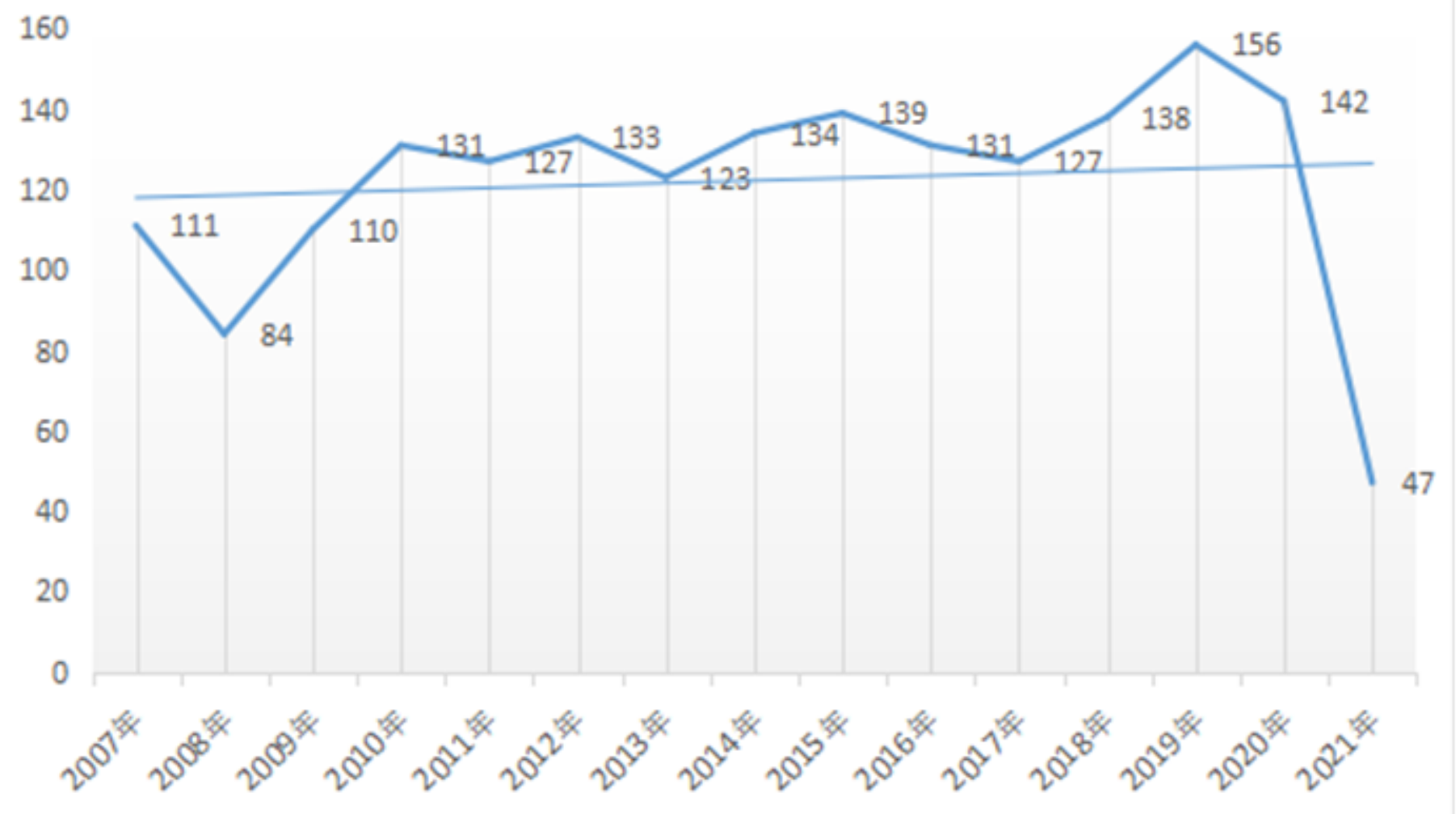

\section{Figure 1}

Characteristics of aerobic exercise research literature in the United States 

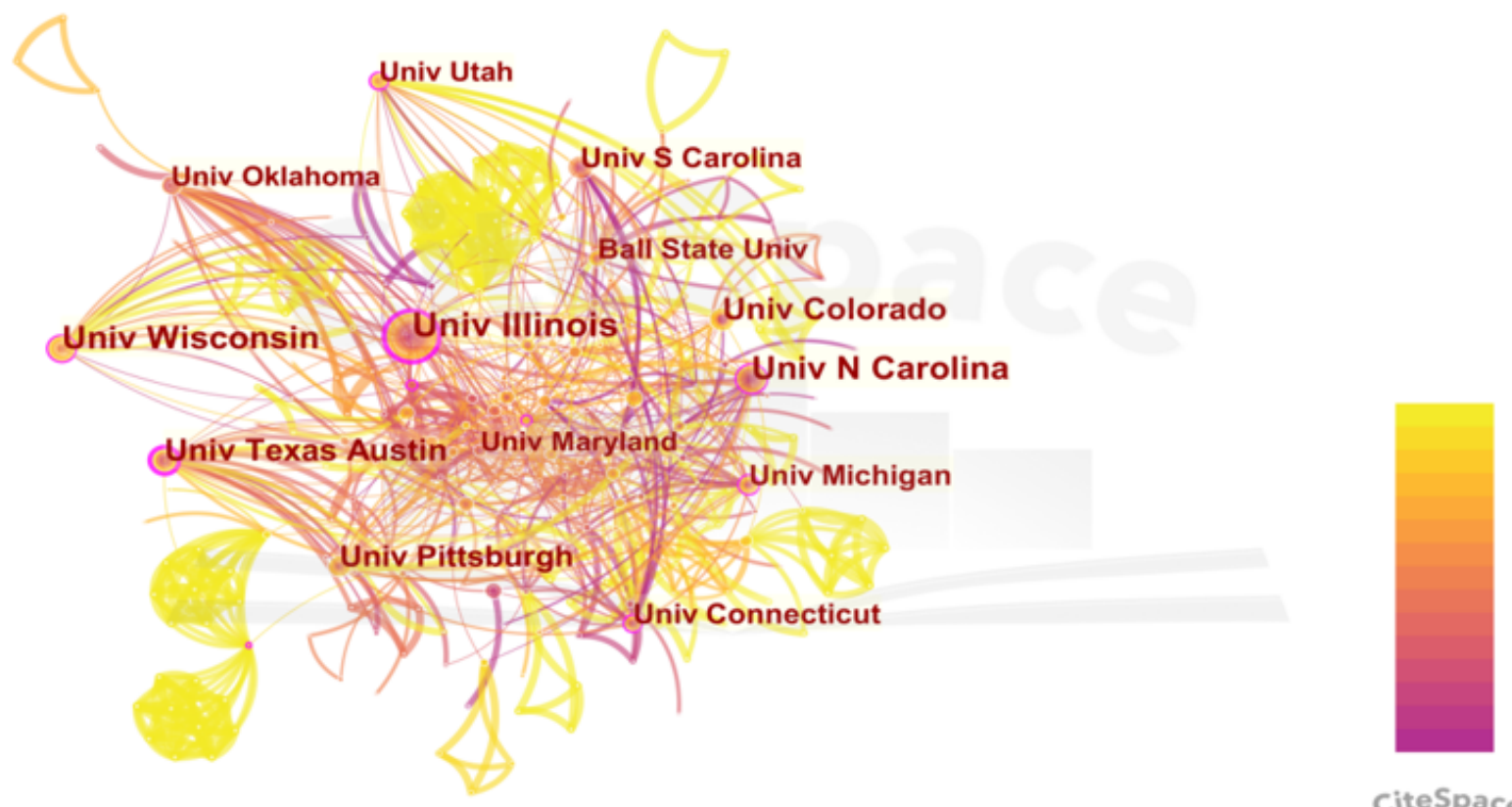

\section{Figure 2}

Co-occurrence network atlas of Major U. S. Aerobics Research Institutions

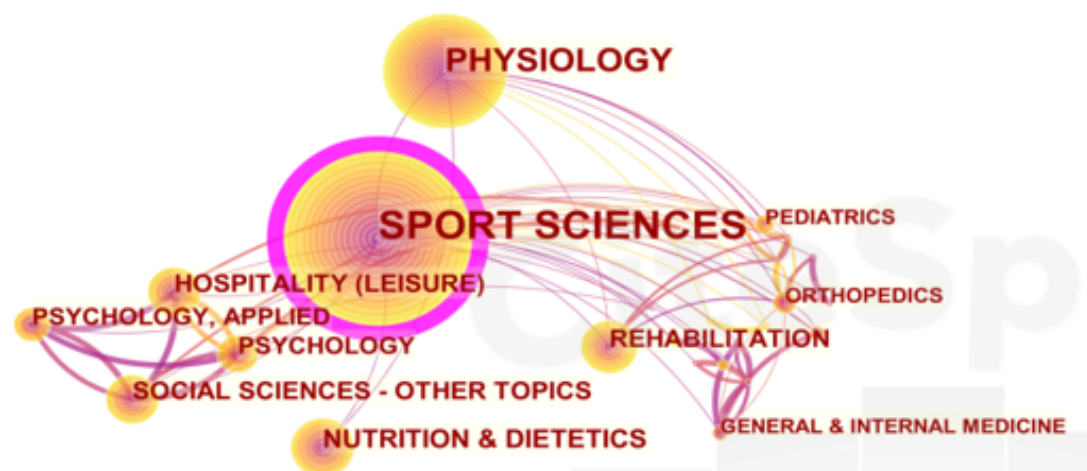

\section{Figure 3}


Co-occurrence network atlas of the major disciplines of aerobic exercise research in the United States

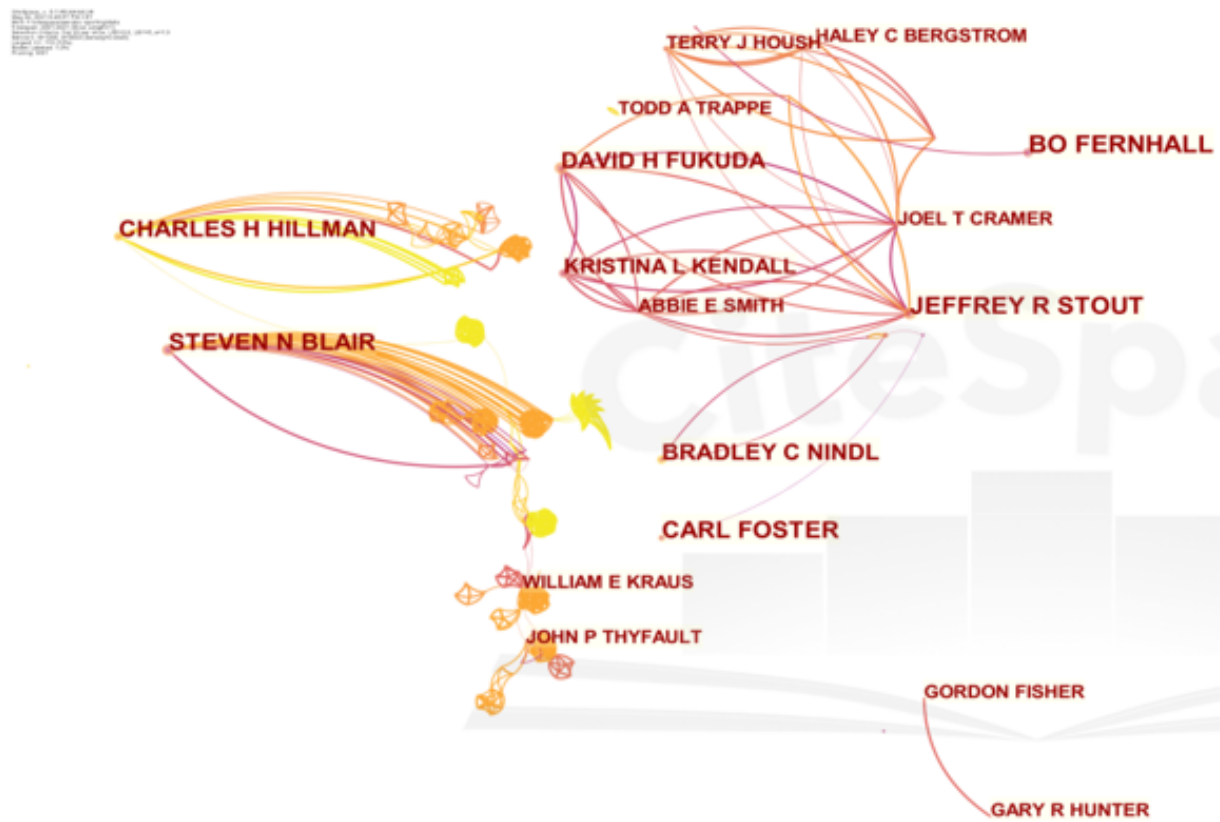

\section{Figure 4}

Aerobic exercise researchers in the United States

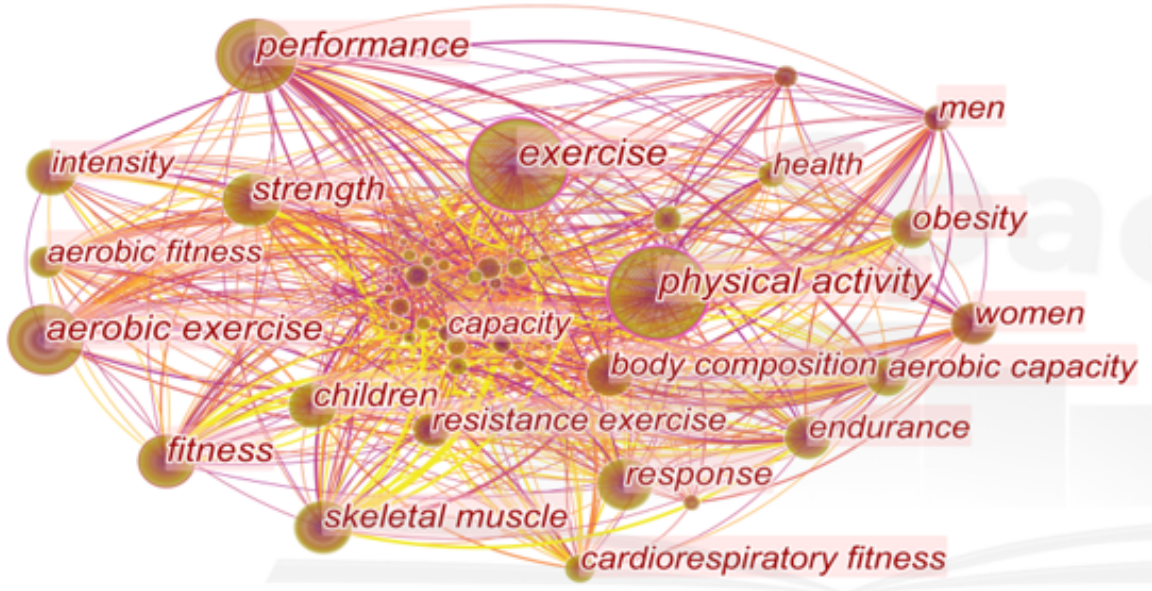

Figure 5 
Network atlas of aerobic exercise research keywords in the United States

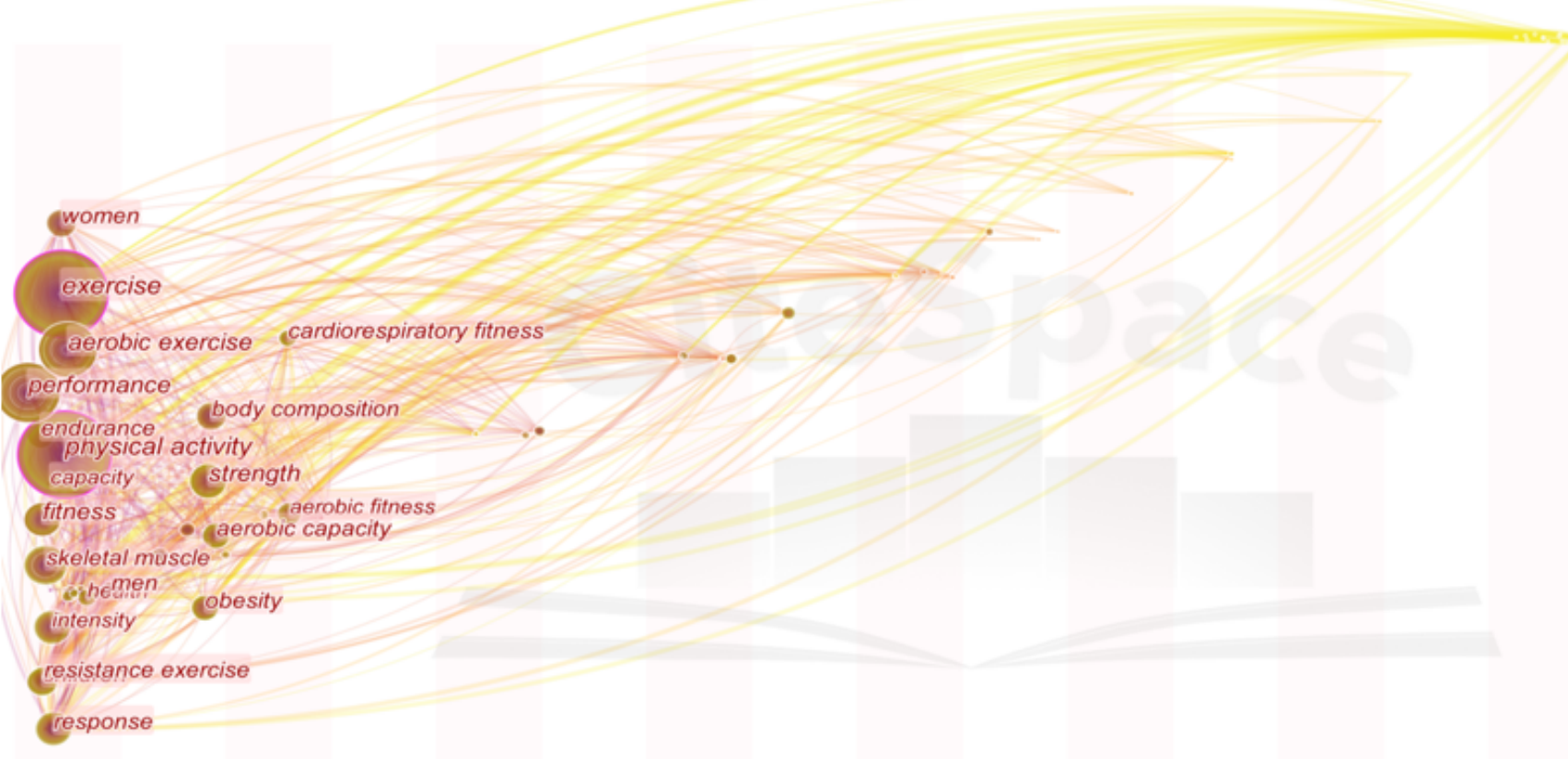

\section{Figure 6}

Time zone network atlas of aerobic exercise research keywords in the United States

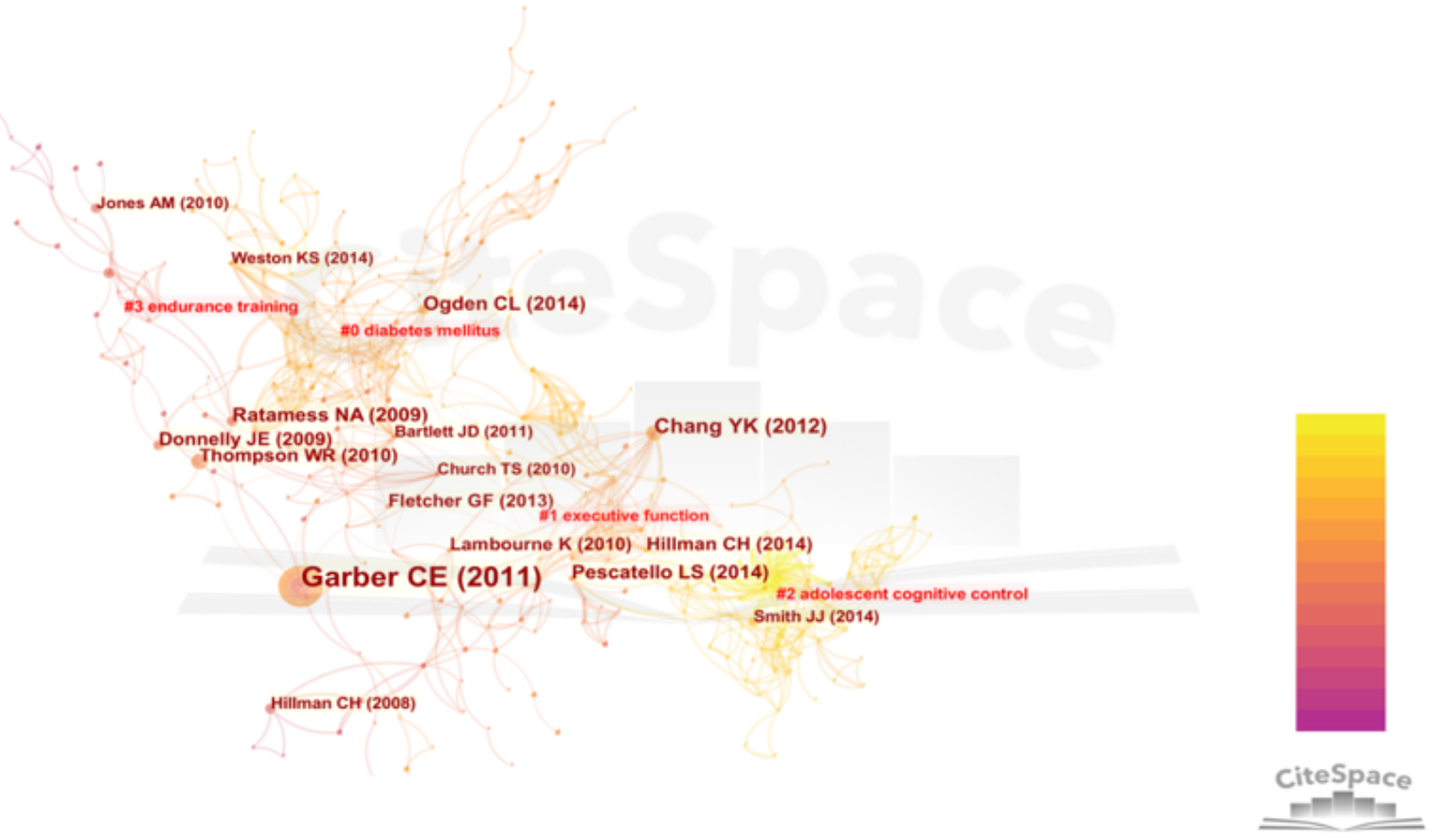


Figure 7

Co-citation atlas of aerobic exercise research literature in the United States 